\title{
LA FINANCIACIÓN DE
}

NUEVAS EMPRESAS EN COLOMBIA, UNA MIRADA DESDE LA PERSPEGTIVE FROM DEMANDA

FECHA DE RECEPCIÓN: 14 de enero FECHA DE APROBACIÓN:10 de marzo Pp. 118-131

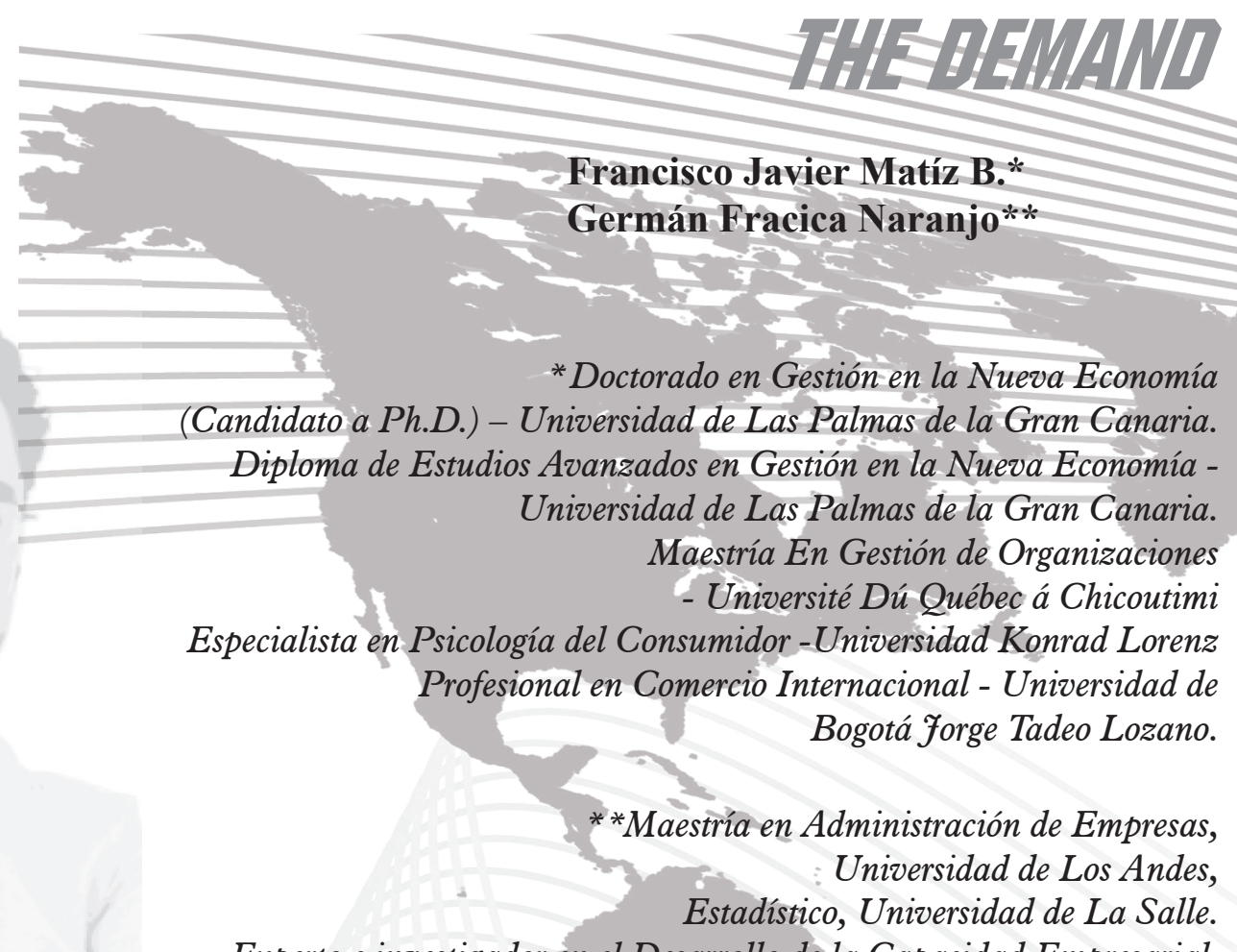

Experto e investigador en el Desarrollo de la Capacidad Empresarial,

la creación de empresas, la Pyme y el e-Business.

Director de las áreas Financiera y Cuantitativa en la Universidad Externado de Colombia.

Fundador y director del programa de Gerencia para la

Pequeña y Mediana Empresa en las universidades Externado de Colombia y de La Sabana. Profesor de posgrado. Asesor gerencial en pequeña y mediana empresa. Par académico, evaluador reconocido por Colciencias en proyectos de investigación sobre pequeña y mediana empresa, creación de empresas, desarrollo de la capacidad empresarial, así como jurado evaluador de planes de negocio en el concurso Ventures. 


\section{RESUMEN}

El Emprendimiento y la creación de empresas se han convertido en una de las principales estrategias para la definición de políticas enfocadas al desarrollo socioeconómico, sin embargo una de las más comunes barreras para el fomento y desarrollo empresarial se encuentra en la consecución por parte de los emprendedores y nuevos empresarios de los recursos financieros necesarios para la consolidación de sus proyectos; durante los últimos años a nivel mundial se han venido consolidando diferentes propuestas y esquemas para la solución de este tipo de situaciones con la generación de alternativas de capital de riesgo, las cuales para el caso colombiano se encuentran en etapa de desarrollo.

Este artículo presenta algunos de los resultados del Proyecto de Investigación "Fuentes de Financiación para Start Ups” realizado por los grupos de Investigación Entrepreneurship (Universidad EAN) y Cultura Emprendedora (Universidad de la Sabana), con el apoyo del Departamento Administrativo de Ciencia, Tecnología e Innovación Colciencias, reflejando las características, necesidades y percepciones de los emprendedores ante la financiación de sus iniciativas y proyectos empresariales.

\section{ABSTRACT}

Entrepreneurship and company creation have turned to be one of the principal strategies to define the existing policies focused on socio-economic development; however, one of the commonest barriers of entrepreneurial consolidation and development is based on the actual use of resources by entrepreneurs and new-born company managers to conclude their projects. In the last few years, at world level, different proposals and frameworks have been made to solve problems generated by alternatives of risk capital, which are actually in a developmental stage in Colombia.

This article shows some of the research project's results made by EAN Entrepreneurship research group and Sabana university Entrepreneurial culture group, with the support of Colciencias science, technology and innovation administrative department, showing characteristics, needs and perceptions of entrepreneurs when dealing with the financing of entrepreneurial initiatives and projects.

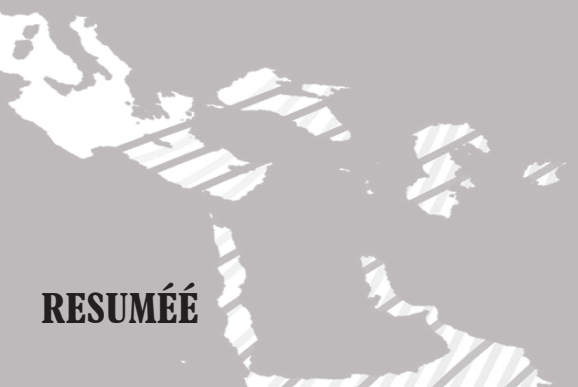

L'entrepreneuriat et la création d'entreprises se sont convertis en l'une des principales stratégies pour définir des politiques tournées vers le développement socio-économique d'une région, mais. cependant, un des obstacles les plus récurrents auquel sont confrontés les entrepreneurs lors de la création et du développement de leur entreprise est le problème de l'accès aux ressources financières nécessaires pour la consolidation de leurs projets. Au cours des dernières années et au niveau mondial, différentes propositions et solutions pour la résolution de telles situations ont été mises en cuvre grâce à la génération de solutions alternatives de capital de risque, qui, dans le cas de la Colombie, n'en sont qu'au stade de développement.

Cet article présente certains des résultats du travail "Sources de financement pour Start Ups ", projet de recherche réalisé par les groupes Entrepreneurship (Université EAN), Culture Entrepreneuriale (Université de la Sabana), avec le soutien du département de Science Administrative, de la technologie et de l'innovation de Colciencias. Cet article met en lumière les caractéristiques, les besoins et les perceptions des entrepreneurs pour le financement de leurs initiatives et projets d'entreprise. $\rightarrow$ Palabras claves

Emprendimiento Creación de empresas

Financiación de nuevas empresas

Venture capital $\rightarrow \quad$ Key words

Entrepreneurship

Company creation

New company financing

Venture capital

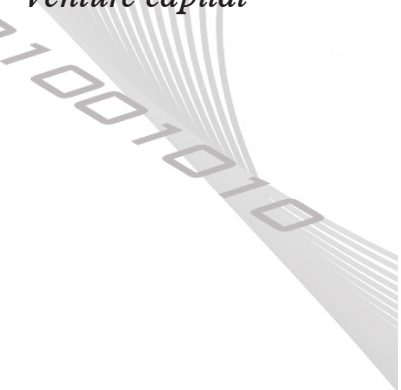

Esprit d'entreprise

Financement de

nouvelles entreprises

Capital risque 


\section{INTRODUCcIón}

1 emprendimiento y la creación de empresas se han convertido en un movimiento realmente importante en el desarrollo socioeconómico de la sociedad post moderna. En la actualidad los diferentes gobiernos y las mismas entidades multilaterales no dudan en reconocer públicamente su importancia en la generación de desarrollo y bienestar de cualquier economía, un ejemplo de ello es el hecho de que la Unión Europea desde el año 2000 haya planteado un plan de reformas en el que se incluyó entre otras cosas la puesta en marcha de la Carta Europea de la pequeña empresa. De esta manera se genera el "Libro Verde sobre el espíritu Empresarial" que pone sobre la mesa de la comunidad europea el tema de los emprendedores y cómo generar programas y acciones que permitan su crecimiento (Comisión de las Comunidades Europeas, 2003).

El emprendedor como tema de investigación es una disciplina aún joven (Vecina 1999, Cooper 2003, Matiz 2009) y el término en sí, se debe a la acepción proveniente del vocablo francés Entrepreneur, utilizado por primera vez por Cantillon durante la primera mitad del Siglo XVIII, con la intención de identificar a aquel individuo que tomaba la responsabilidad de poner en marcha y llevar a buen término un proyecto, desde ese momento y a lo largo de la historia las diferentes escuelas económicas se han referido de una y otra forma al emprendedor, desde la visión clásica del emprendedor como un trabajador superior y la neoclásica que le otorgaba un rol de coordinador, hasta llegar a la escuela austriaca que lo supone como el eje del desarrollo económico.

En la actualidad y gracias a los planteamientos entre otros de Schumpeter, el emprendedor se define como un individuo innovador, que identifica y crea nuevas oportunidades de negocio, para lo cual debe reunir y combinar diferentes recursos que le permiten maximizar los beneficios provenientes de sus innovaciones, todo esto en un entorno caracterizado por la incertidumbre (Amit et al., 1993).

Las aproximaciones al emprendimiento como tema de estudio se han dado entonces desde variados y diferentes enfoques, ya que este sería claramente deficiente si se concentrara a estudio únicamente de las características y comportamientos de los empresarios como individuos (Van de Ven, 1993); es por ello que se han tenido en cuenta diferentes factores asociados al análisis del emprendedor y su accionar, factores tales como los sociales, los estructurales y los económicos han sido abordados para el estudio del emprendedor y el proceso de la creación de empresas.

Uno de los temas de mayor interés a la hora de estudiar el emprendimiento como fenómeno socioeconómico ha sido la identificación de las principales variables que afectan el proceso de creación de empresas enfocándose en los factores claves de éxito o en las principales problemáticas enfrentadas por los emprendedores a lo largo de su proceso empresarial, frente a este tópico Kantis y Otros (2002) buscaron identificar las principales proble-máticas percibidas por emprendedores argentinos discriminándolos de acuerdo a su nivel de formación como emprendedores de Base Universitaria (EBU) y emprendedores no Universitarios (ENU), encontrando que claramente sin importar los niveles de formación los dos principales problemas a los que se enfrentan los nuevos empresarios están ligados al acceso a los mercados (Obtener Clientes) y la financiación de sus iniciativas empresariales (financiar y manejar el flujo de caja) tal como se presenta en la Tabla $\mathrm{N}^{\circ} .1$, a partir de la generalización de estas problemáticas no sólo en las nuevas empresas, sino en general en el sector productivo de los diferentes países encuentra en la financiación empresarial una problemática de gran importancia. 
Tabla 1. Problemática en la creación de empresas

\begin{tabular}{|l|r|r|}
\hline \multicolumn{1}{|c|}{ PRINCIPAL PROBLEMA } & EBU & ENU \\
\hline Obtener información del mercado & $31 \%$ & $26 \%$ \\
\hline Obtener clientes & $72 \%$ & $61,5 \%$ \\
\hline Conseguir proveedores adecuados & $44 \%$ & $51 \%$ \\
\hline Contratar gerentes & $16 \%$ & $13 \%$ \\
\hline Contratar trabajadores calificados & $52 \%$ & $49 \%$ \\
\hline Tener el equipamiento adecuado & $41 \%$ & $61,5 \%$ \\
\hline Gerenciar la empresa & $44 \%$ & $28 \%$ \\
\hline Financiar y manejar el flujo de caja & $68 \%$ & $67 \%$ \\
\hline Manejar las operaciones de la empresa & $32 \%$ & $41 \%$ \\
\hline Certificar estándares de calidad & $30 \%$ & $23 \%$ \\
\hline $\begin{array}{l}\text { Adaptar el producto/servicio a las } \\
\text { necesidades del mercado }\end{array}$ & $42 \%$ & $44 \%$ \\
\hline $\begin{array}{l}\text { Manejar las relaciones con grandes } \\
\text { clientes }\end{array}$ & $37 \%$ & $36 \%$ \\
\hline
\end{tabular}

Fuente. Kantis et al, (2002)

Colombia no es la excepción y si bien durante los últimos años el fenómeno emprendedor ha tenido una importante dinámica en diferentes campos, tales como la formación de emprendedores, la generación de políticas y programas de apoyo al emprendimiento y sobre todo el desarrollo de un importante número de iniciativas de apoyo, tanto financiero como no financiero, de origen público como privado, la financiación de las iniciativas del cada vez mayor número de emprendedores es un tema de gran relevancia.

De acuerdo con los Informes anuales del Global Entrepreneurship Monitor (GEM), la principal investigación en temas de emprendimiento a nivel mundial en la cual Colombia participa desde el año 2006, Colombia se ha venido presentando como uno de los países con mayores niveles de emprendimiento en el mundo lo que se refleja en que de acuerdo con estos estudios, en el país existen más de seis millones de emprendedores en
Durante los últimos años el

fenómeno emprendedor ha tenido una importante dinámica en diferentes campos tales como la formación de emprendedores, la generación de políticas y programas de apoyo al emprendimiento y sobre todo el desarrollo de un importante número de iniciativas de apoyo tanto financiero como no financiero, de orígen público como privado, la financiación de las iniciativas del cada vez mayor número de emprendedores es un tema de gran relevancia. la ardua tarea de consolidar sus propuestas empresariales; sin embargo estas iniciativas o proyectos están aún lejos de ser mayoritariamente propuestas innovadoras y sostenibles en el tiempo, situación que se ve claramente reflejada a la hora de indagar sobre los niveles de inversión involucrados en las mismas, tal como se puede observar en la tabla 2, los promedios de inversión de las nuevas empresas colombianas no son muy superiores a los $\$ 30$ millones de pesos (US\$15500 aprox.), una cifra que sumada a los valores tanto de la moda como la mediana de la muestra (\$10 millones ó US\$ 5000 aprox.) denotan claramente la orientación del país hacia la generación de emprendimiento principalmente de necesidad, modelos de negocio básicos con bajas propuestas de valor agregado y limitados niveles de innovación o apropiación tecnológica.

Tabla 2. Financiación de nuevas empresas

\begin{tabular}{|l|r|r|r|r|}
\hline \multicolumn{1}{|c|}{$\begin{array}{c}\text { Financiación de } \\
\text { nuevas empresas }\end{array}$} & \multicolumn{1}{c|}{$\mathbf{2 0 0 6}$} & \multicolumn{1}{c|}{$\mathbf{2 0 0 7}$} & \multicolumn{1}{c|}{$\mathbf{2 0 0 8}$} & \multicolumn{1}{c|}{$\mathbf{2 0 0 9}$} \\
\hline Promedio de inversión requerida (pesos) & 23.655 .783 & 24.355 .444 & 32.583 .950 & 31.070 .965 \\
\hline Moda (pesos) & 5.000 .000 & 5.000 .000 & 10.000 .000 & 10.000 .000 \\
\hline Mediana (pesos) & 10.000 .000 & 5.000 .000 & 10.000 .000 & 10.000 .000 \\
\hline$\%$ de casos por debajo del promedio & $78.00 \%$ & $76.00 \%$ & $83.00 \%$ & $80.90 \%$ \\
\hline
\end{tabular}

Fuente. GEM Colombia (2009) 
Es entonces a partir de esta situación que los grupos de investigación Entrepreneurship Group y Cultura Emprendedora de las Universidades EAN y Sabana respectivamente, con el ánimo de generar un aporte significativo desde la investigación al conocimiento y reflexión sobre la dinámica empresarial, durante los años 2009 y 2010 desarrollaron el proyecto de investigación "Fuentes de
Financiación para Empresas en Etapa de Start Up", una iniciativa que contó con el apoyo y la financiación del Departamento Administrativo de Ciencia Tecnología e Innovación, Colciencias, y dentro del cual como una de sus partes se abordó el estudio de la demanda de recursos financieros para la creación de empresas del cual surge como uno de sus productos el presente artículo.

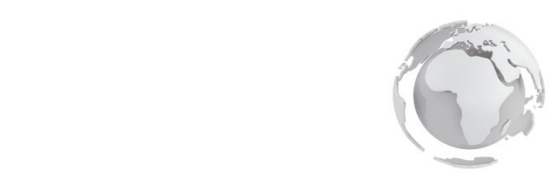

2. MARCO TEÓRICO

n factor no solo predominante en el proceso de desarrollo empresarial sino fundamentalmente constante, es la financiación de las organizaciones que lo asumen, esto, debido a que a lo largo de su proceso de evolución y desarrollo desde la concepción misma de

la iniciativa, hasta la consolidación y crecimiento de esta, el acceso a recursos

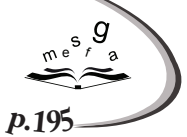
entre ellos los financieros, es una necesidad constante y en evolución; es así como en cada una de estas etapas se han desarrollado diferentes modelos de financiación diseñados teniendo en cuenta las características tanto de las organizaciones como de sus gestores, generando un esquema denominado la cadena de la financiación empresarial (Matiz y Mogollón 2008).

Cardullo (1999) plantea esta cadena de la financiación empresarial en una clara relación entre las diferentes fuentes o mecanismos de financiación y los niveles de desarrollo de las empresas beneficiarias de los mismos, de esta manera en la medida en que una empresa evoluciona en el proceso de desarrollo empresarial, sus mecanismos y estrategias de financiación se hacen a su vez cada vez más especializadas tal y como se presenta en el figura 1.

Figura 1. Financiación y desarrollo empresarial

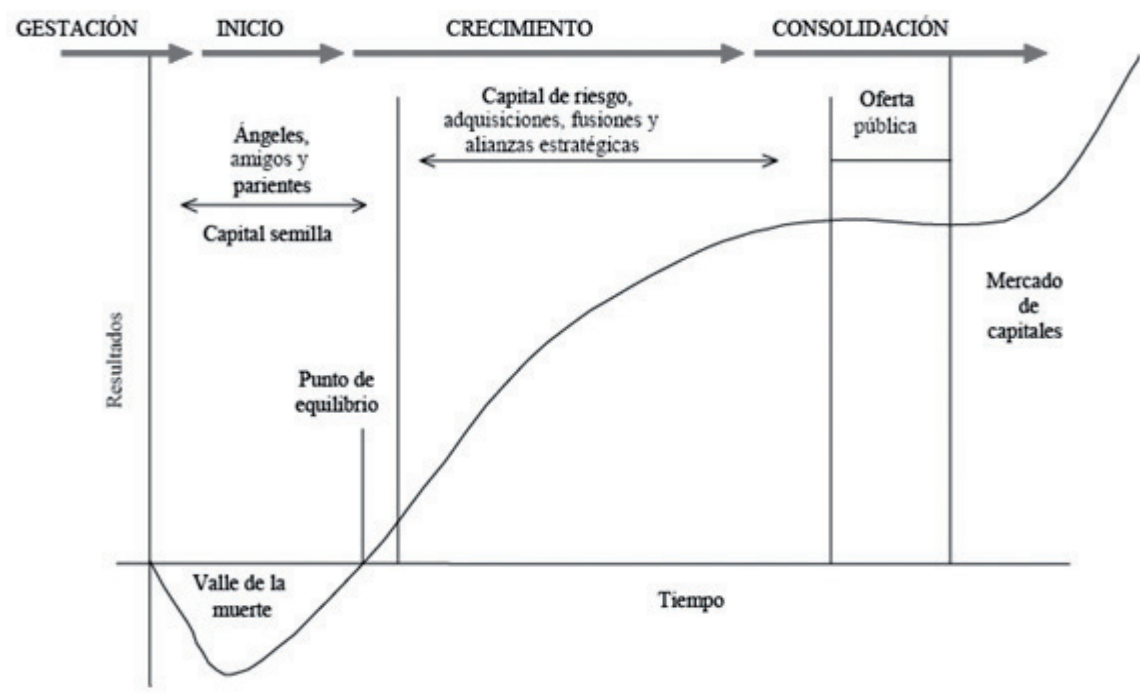

Fuente. Cardullo (1999). 
Otros autores como Berger y Udell (1998) sostienen que las PyMEs que tienen una estrategia de crecimiento recorren un ciclo financiamiento en el que acceden a fuentes e instrumentos a medida que las empresas crecen, ganan experiencia y a su vez el proceso se hace más transparente. A medida que la empresa crece puede acceder a financiamiento a través de intermediarios, capital de riesgo y préstamos bancarios. Finalmente, aquellas empresas que sobreviven y continúan creciendo acceden a los mercados públicos. (Matiz y Mogollón 2008)

El casocolombianose alejaun poco de estos planteamientos, en gran medida porque el mercado financiero para el emprendimiento o las nuevas empresas es un ejercicio aún incipiente y en etapas realmente tempranas; históricamente el país ha abordado la financiación empresarial como el resultado de esfuerzos únicamente del sector bancario de allí la explicación de gran parte de la problemática que da origen al presente estudio. De acuerdo con Arbeláez, Zuleta y Velasco (2004), el 14\% de los créditos solicitados por las microempresas colombianas es rechazado principalmente por problemas tales como la Incapacidad de pago de los solicitantes (20\%), la no presentación de ingresos permanentes $(15 \%)$, así como problemas a la hora de presentar codeudores $(28 \%)$, o por encontrase reportados en centrales de riesgo (5\%), situaciones que en gran medida menoscaban las reales

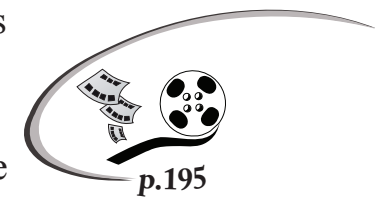
posibilidades de desarrollo económico del sector pyme y emprendedor en el tejido empresarial colombiano.

Mercados más desarrollados como lo son el de los Estados Unidos o Europa, presentan una Industria de Capital de Riesgo con una gran variedad de inversionistas tanto institucionales como particulares (Barona, Gómez y Torres 2006), situación que en gran medida ha generado el desarrollo de nuevas iniciativas a lo largo del mundo, siendo Colombia uno de los muchos ejemplos que de una manera más reciente se han preocupado por la generación de una mayor, aunque aún no suficiente, oferta de mecanismos para la financiación de nuevas empresas; sin embargo esta incipiente industria se presenta aún alejada del grueso del movimiento emprendedor del país siendo esta una de las principales motivaciones para el desarrollo del presente artículo.

\section{。 \\ 3. METOdOLOGÍA}

1 ara el desarrollo de la presente investigación sobre la Financiación de Nuevas Empresas en Colombia, se desarrolló un ejercicio exploratorio desde la perspectiva de los potenciales empresarios (La Demanda), para lo cual se diseño un estudio cuantitativo cuyo objetivo principal se orientó a conocer la percepción de los emprendedores colombianos frente a la financiación de sus empresas, investigando igualmente algunas características significativas tanto de su perfil como del de sus proyectos empresariales.

El cálculo la población objetivo se tuvo teniendo en cuenta la caracterización utilizada por el Global Entrepreneurship Monitor(GEM) comoel principal ejerciciode investigación en emprendimiento a nivel mundial, en este orden de ideas, la población objetivo está conformada por adultos que se encuentran en el rango de edad comprendido entre los 18 y 64 años y que se encuentran adelantando el proceso de planeación y desarrollo de propuestas empresariales.

El canal de acceso a la población objetivo y la aplicación del instrumento diseñado para el estudio, fue el llegar a emprendedores vinculados a programas y proyectos de apoyo a emprendedores tales como Universidades (Universidad EAN, Pontificia Universidad Javeriana sede Cali, Universidad EAFIT), el Programa Bogotá Emprende de la Cámara de Comercio de Bogotá y la Alcaldía Mayor de Bogotá D.C., el Programa Parque E., de la Universidad de Antioquia y la Alcaldía de Medellín, así como los nodos de incubación de la Incubadora de Empresas Créame; entidades a las que los autores agradecen su colaboración y compromiso. 
Para el cálculo del tamaño de la muestra se trabajó como valor total de la población, el número de emprendedores nacientes arrojado por el informe GEM Colombia 2008, el cual corresponde a 3.703 .746 personas.

Tabla 3. Tasa de actividad emprendedora (TEA) 2008

\begin{tabular}{|l|c|c|c|c|r|}
\hline \multicolumn{1}{|c|}{ Tipo de iniciativas } & $\begin{array}{c}\text { Estimación } \\
\mathbf{2 0 0 6}\end{array}$ & $\begin{array}{c}\text { Estimación } \\
\mathbf{2 0 0 7}\end{array}$ & $\begin{array}{c}\text { Estimación } \\
\mathbf{2 0 0 8}\end{array}$ & $\begin{array}{c}\text { Variación } \\
\text { porcentual } \\
\mathbf{2 0 0 6 - 0 7}\end{array}$ & $\begin{array}{c}\text { Variación } \\
\text { porcentual } \\
\mathbf{2 0 0 7 - 0 8}\end{array}$ \\
\hline Emprendedores nacientes (0-3 meses) & 2.808 .672 & 2.103 .330 & 3.703 .746 & $-34 \%$ & $43 \%$ \\
\hline Nuevos empresarios (3-42 meses) & 3.227 .915 & 4.072 .907 & 3.143 .628 & $21 \%$ & $-30 \%$ \\
\hline Total (0-42 meses) & 5.781 .955 & 5.958 .561 & 6.571 .335 & $3 \%$ & $9 \%$ \\
\hline
\end{tabular}

Fuente. GEM Colombia, 2008. Encuesta a la población adulta.

De estas cifras vale la pena destacar, como ya se ha mencionado anteriormente, la tendencia creciente de la población colombiana involucrada en procesos de desarrollo empresarial, situación esta que ha consolidado durante los últimos años
(2006 - 2009) al país entre los que presentan mayores niveles de tasa de actividad emprendedora a nivel mundial.

Es entonces a partir de los datos arrojados por el GEM 2008, que se planteó el ejercicio estadístico para la determinación de la muestra del presente estudio, utilizando un muestreo aleatorio de acuerdo con las siguientes condiciones:

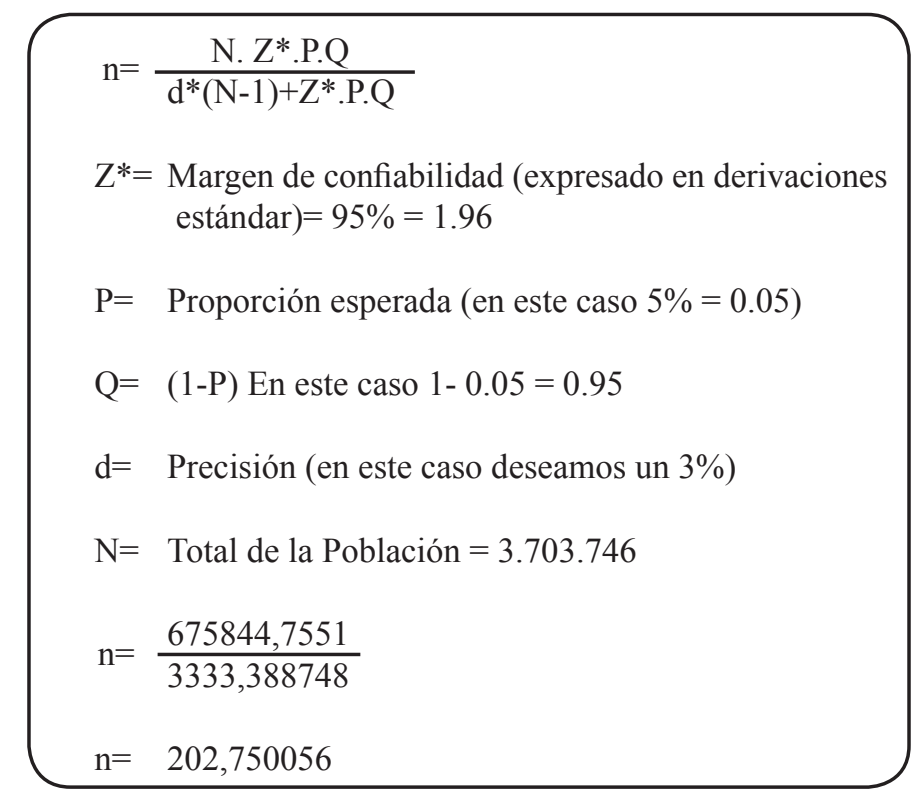




\section{RESULtAdos}

omo resultado del trabajo de campo y en concordancia con los objetivos del estudio, se logró inicialmente contar con la caracterización de los emprendedores o futuros empresarios que hicieron parte de la muestra, la cual se puede contrastar con información como la suministrada por los Informes GEM Colombia de los últimos años, en este sentido mientras el GEM presenta un amplia participación de emprendedores hombres por encima de las mujeres a la hora de crear empresa, la muestra de este estudio fue mucho más homogénea en cuestión de género

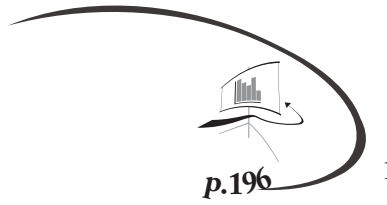

ya que el $52 \%$ de los encuestados fueron mujeres, frente a un $48 \%$ de hombres; por otra parte en lo que se refiere a la variable edad la gran mayoría de los encuestados está ubicada en los dos primeros quintiles de la población, es decir entre los 18 y 39 años, situación completamente coherente con la distribución presentada por el GEM, en el que las mayores TEA (Tasas de Actividad Emprendedora) se encuentran en los rangos de 25 a 34 y 35 a 44 años respectivamente.

Finalmente y para terminar de caracterizar a la muestra representativa de la demanda de recursos financieros para la creación de empresas en Colombia, el presente estudio indagó sobre los niveles de formación, variable que claramente ha sido definida como una determinante de éxito en los procesos empresariales (Honig, 1998; Basu y Goswami, 1999; Van Praag y Cramer, 2001; Watson et al., 2003; Bosma et al., 2004; Collins-Dodd et al., 2004), aunque para algunos autores los mayores niveles de formación académica se conviertan en un riesgo para las iniciativas empresariales, en la medida en que se convierten en mayores posibilidades de movilidad laboral que afectan directamente la persistencia de los emprendedores (Gimeno et al., 1997). De esta manera el $75 \%$ de los encuestados hacen parte de lo que algunos autores han denominado los emprendedores de base universitaria (Postigo 2002), es decir aquellos que cuentan con formación universitaria, y que para este estudio están representados principalmente por profesionales universitarios en un $40 \%$ y por profesionales con estudios de postgrado en un $35 \%$, seguidos por un $17 \%$ de los encuestados que cuentan con niveles de formación técnica y tecnológica. Datos estos acordes con los presentados por el GEM, donde se menciona que existe "una relación directa entre mayor nivel educativo y mayor nivel de actividad emprendedora".

Ya entrando en materia y como primera variable a evaluar frente a la situación de los emprendedores colombianos frente a sus necesidades en lo que respecta a los recursos financieros para el desarrollo de sus proyectos, se indagó sobre las necesidades globales de recursos financieros para sus emprendimientos, encontrando una clara necesidad por parte de los mismos frente a fuentes externas de financiación para sus modelos de negocio, ya que el $81 \%$ de los encuestados mencionó no contar con el capital necesario para la creación de sus empresas, frente a 16\% que manifiestan disponer de estos recursos tal y como se observa en la Gráfica $\mathrm{N}^{\circ}$ 2, este resultado claramente define la situación del emprendimiento colombiano frente a las necesidades de soluciones financieras para los nuevos gestores empresariales, así como determina la pertinencia del presente estudio, toda vez la problemática del acceso a fuentes de financiación empresarial es una amplia necesidad a nivel nacional y como ya se ha mencionado una de las principales variables determinadoras del éxito para la generación de una nueva clase empresarial.

Figura 2. Disponibilidad de recursos financieros

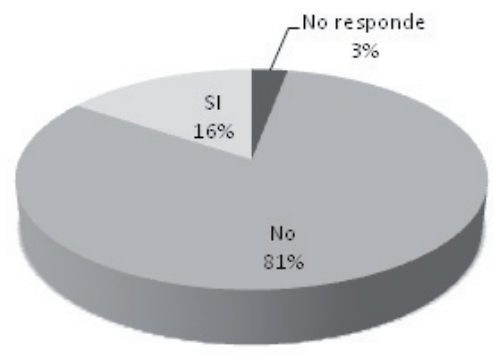


Esta clara tendencia a la necesidad de fuentes de financiación está respaldada por otra parte por el supuesto conocimiento de los emprendedores de cuáles pueden ser las diferentes fuentes que provean los recursos necesarios, ya que el $70 \%$ de ellos mencionaron conocer las fuentes de financiación a las que podrían recurrir, frente a un menor pero nada despreciable $30 \%$ que mencionan no tener conocimiento de cómo acceder a estos recursos; esta situación de un relativo conocimiento en la materia en cuestión fue contrastada con la realidad al indagar posteriormente sobre la referencia a instrumentos o fuentes de financiación para nuevas empresas de manera mucho más específica.

\section{Figura 3. Conocimiento de fuentes de financiación}

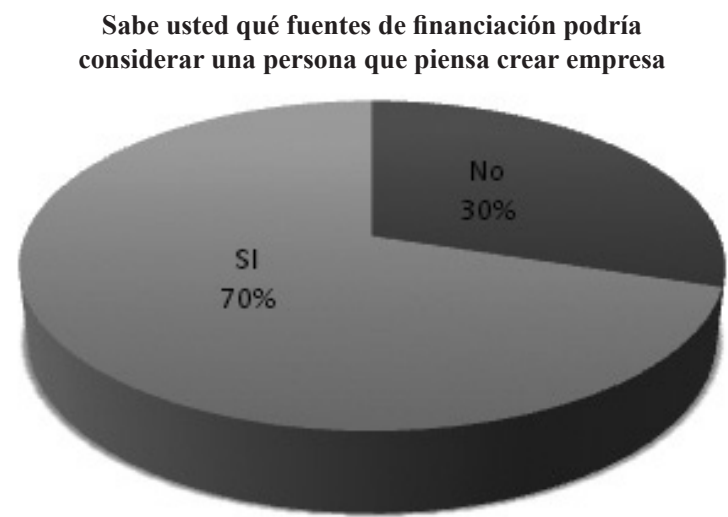

Fuente. Elaboración propia

El hecho de que la gran mayoría de emprendedores no cuenta con una amplia información o conocimiento de las diferentes fuentes de financiación para sus iniciativas empresariales se hace evidente cuando como ya se había mencionado, se indaga sobre la información de que disponen de herramientas o instrumentos de manera mucho más específica, tal y como se podría suponer son las fuentes que se podrían denominar tradicionales como lo es el financiación bancaria (créditos), los recursos propios o el financiamiento a través de particulares (préstamos familiares y de amigos) las de mayor referencia representando cifras del $64 \%, 41 \%$ y $38 \%$ respectivamente; frente a niveles de conocimiento del $24 \%$ y $21 \%$ de herramientas de financiación mucho más especializadas en la creación de empresas como lo son el capital semilla y la figura de ángeles inversionistas, tal y como se puede observar en la figura 4.

Figura 4. Conocimiento de fuentes de financiación 2

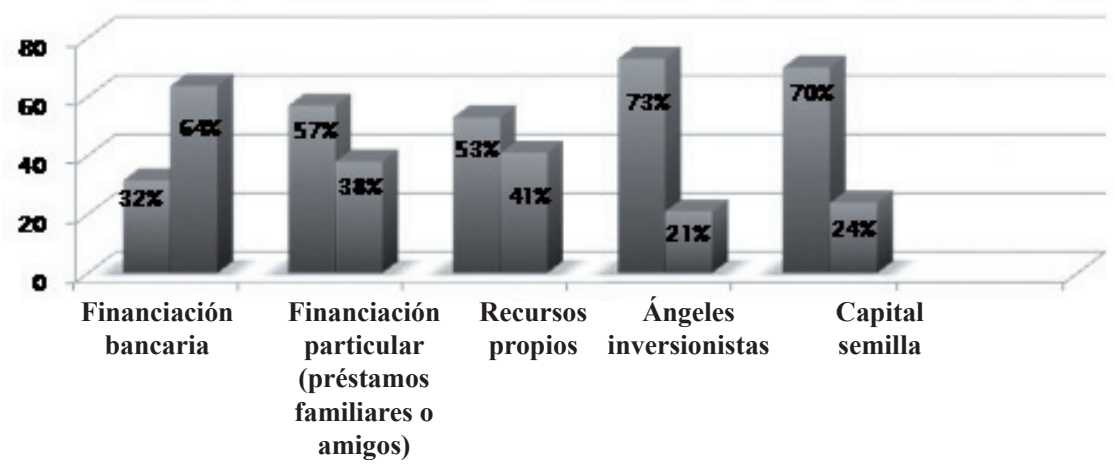

Fuente. Elaboración propia 
Más allá del conocimiento de las fuentes, al indagar sobre cuáles de ellas serían las que harían parte de su estrategia de financiación como la manera de acceder a los recursos necesarios para su proyecto empresarial, y en clara relación con sus manifestaciones de información o conocimiento sobre el tema el $67 \%$ se orientó hacia la financiación bancaria, situación que prevé el ya continuo desencanto de los emprendedores frente al sector financiero debido claramente a que la financiación bancaria es una herramienta no diseñada para estos fines, por lo que repetidamente sus representantes han manifestado su poco o nulo interés frente a los procesos de creación de empresas.
Por otra parte estos resultados se convierten en un punto de reflexión para la gran cantidad de entidades y programas de apoyo financiero y no financiero a emprendedores que se ha ido desarrollando a nivel nacional en lo que se ha venido denominando la "Industria Soporte del

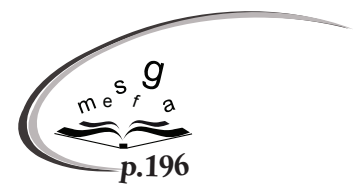
Emprendimiento", ya que estos niveles de desconocimiento por parte de los emprendedores no son otra cosa que una alarma para la generación de estrategias de comunicación que permitan el mejor uso de las alternativas existentes.

\section{Figura 5. Estrategia de financiación}

\section{Como pretende conseguir el capital necesario para crear su empresa}

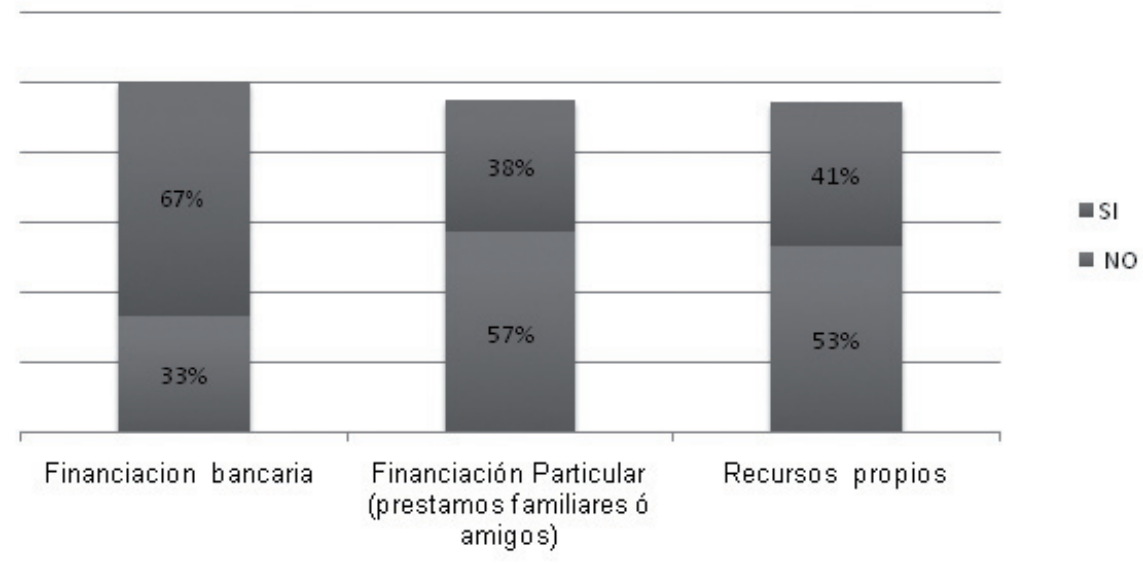

Fuente. Elaboración propia

Es claro entonces él por qué los emprendedores colombianos a la hora de argumentar cuales son las principales barreras o trabas a la hora de acceder a la financiación empresarial se refieren a aspectos puramente ligados con el proceso crediticio tales como las altas tasas de interés, la falta de experiencia crediticia o la falta de garantías reales, variables que denotan de manera contundente el hecho de que los nuevos empresarios no solo carecen de información sobre fuentes alternas para el acceso a los recursos financieros, sino que dentro del esquema bancario se limitan al conocimiento de líneas de crédito que por ser mecanismos no especializados, no cuentan con diseños acordes con la utilización de estos recursos y que sumado a las características ya mencionadas de los emprendedores (como por ejemplo su edad), hacen aún más complejo el acceso al sistema bancario.

Es claro entonces el por qué los emprendedores colombianos a la hora de argumentar cuales son las principales barreras o trabas a la hora de acceder a la financiación empresarial se refieren a aspectos puramente ligados con el proceso crediticio, tales como las altas tasas de interés, la falta de experiencia crediticia o la falta de garantías reales. 
Figura 6. Principales trabas para acceder a financiación

\section{¿Qué aspectos considera son las mayores trabas para acceder a la financiación?}

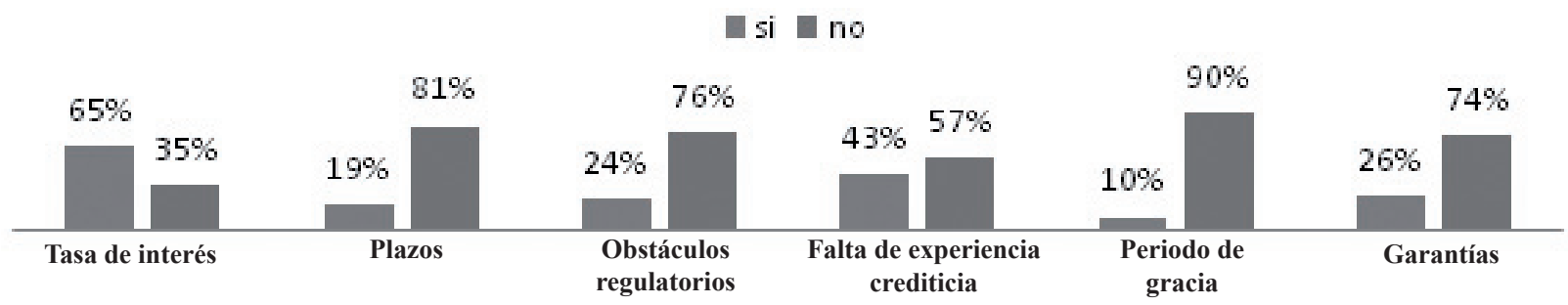

Fuente. Elaboración propia

Finalmente y en contraste con un desconocimiento de fuentes alternativas para la financiación empresarial, y muy contrario a la creencia de que la posición del emprendedor ante la admisión de nuevos socios en sus iniciativas es generalizadamente de rechazo y asociada a una posición egoísta y en algunos casos calificada como de miopía empresarial, los emprendedores indagados manifestaron mayoritariamente estar dispuestos a ceder participación de sus empresas como resultado de un proceso de financiación de las mismas, situación que por una parte demuestra el inicio de la generación de una nueva cultura de inversión en el país, pero fundamentalmente una condición necesaria para que a partir de condiciones de la demanda se pueda

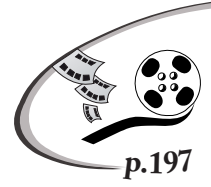
pensar en la estructura y desarrollo de mecanismos de Venture Capital tales como los Ángeles Inversionistas y los Fondos de Capital de Riesgo.

Figura 7. Disponibilidad de ceder participación

\section{¿Estaría dispuesto a ceder participación de su empresa?}

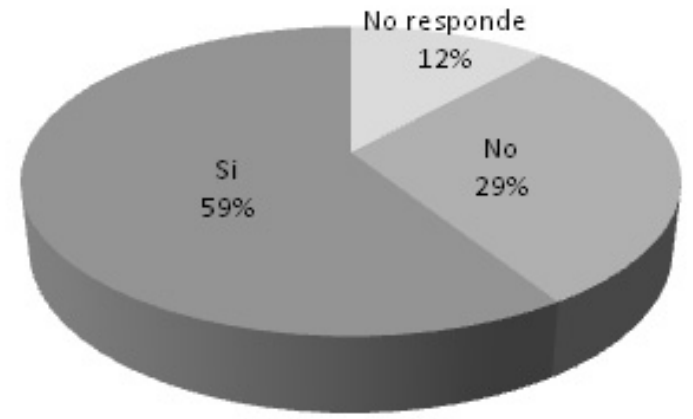

Fuente. Elaboración propia 
Es claro, de acuerdo con los resultados de la presente investigación, que para la gran mayoría de emprendedores colombianos la existencia de una cadena de financiación"y por ende de instrumentos financieros diseñados y ajustados de acuerdo a sus necesidades y las de sus modelos de negocio es aún una propuesta inexistente, los esfuerzos de un importante número de instituciones tanto públicas como privadas durante los últimos años en la generación de la industria del venture capital (Capital de Riesgo) colombiano si bien han sido realmente importantes y han determinado un salto en la evolución de esta temática aún carecen de la difusión necesaria no solo para la formación de un nuevo mercado de inversión, sino para la ruptura de los paradigmas existentes por el emprendedor del común sobre la financiación empresarial.

Sólo en las soluciones crediticias ofertadas por el mercado bancario es que los emprendedores colombianos encuentran una opción alternativa a los recursos propios o de inversionistas informales de sus círculos cércanos, instrumentos financieros que se caracterizan principalmente por ser productos no especializados, diseñados para esquemas de consumo y no para las necesidades de un proceso empresarial, de allí que desafortunadamente en la mayoría de los casos se conviertan en un factor más de las múltiples dificultades a las que debe enfrentarse el emprendedor y su nueva empresas para superar la etapa de puesta en marcha de la misma.
El reto ahora está no sólo en que la industria soporte del emprendimiento en Colombia, debe enfocarse en la generación de alternativas de financiación que aborden instrumentos tales como las redes de Ángeles Inversionistas o los Fondos de Capital de Riesgo, pues en la actualidad existen algunas de estas iniciativas en proceso de desarrollo; sino mucho más allá de ello en la formación de los emprendedores a través de aquellos programas de acompañamiento en temas relacionados por nuevas estrategias de financiación que incluyan estas nuevas propuestas así como esquemas mucho más enfocados a las necesidades empresariales tales como el factoring o el leasing .

La especialización de las fuentes de financiación será entonces una muy probable tendencia en el mercado colombiano tal y como se ha presentado en otros países, y la industria del venture capital deberá poco a poco trascender de aquellos esquemas de Fondos de Capital Privado actuales enfocados más en el crecimiento y consolidación de medianas empresas, a la generación de nuevos fondos mucho más enfocados a capital semilla, lo que si bien implica mayores niveles de riesgo a su vez representará igualmente más rentabilidad para los inversionistas por una parte y por otra un salto en la generación de nuevas empresas, las cuales como condición fundamental para tal fin día a día deberán estar más enfocadas a propuestas soportadas en la identificación de oportunidades de mercado, innovadoras y con cada vez mayores niveles de apropiación y desarrollo tecnológico. 


\section{REFERENCIAS BIBLIOGRÁFICAS}

Basu, A. y Goswami, A. (1999). Determinants of South Asian entrepreneurial growth in Britain: a multivariate analysis. Small Business Economics

Berger, Allen N. y Udell, Gregory F. (1998). The Economics of Small Business Finance: The Roles of Private Equity and Debt Markets in the Financial Growth Cycle. Journal of Banking and Finance.

Cardullo, Mario (1999). Technological Entrepreneurism, Research Studies Press.

Collins-Dodd, C.; Gordon, I.M. y Smart, C. (2004). Further evidence on the role of gender in financial performance. Journal of Small Business Management.

Gimeno, J. Folta, T. Cooper, A. y Woo, C. (1997). Survival of the fittest? Entrepreneurial human capital and the persistence of underperforming firms. Administrative Science.

Global Entrepreneurship Monitor - Informe Colombia - 2006.

Global Entrepreneurship Monitor - Informe Colombia - 2007

Global Entrepreneurship Monitor - Informe Colombia - 2008

Global Entrepreneurship Monitor - Informe Colombia - 2009

Honig, B. (1998). What determines success? Examining the human, financial, and social capital of Jamaican microentrepreneurs. Journal of Business Venturing,

Matiz Francisco (2006). Emprendimiento Como Un Pilar para La Competitividad de las Naciones. Revista EAN.

Matiz Francisco (2009), Investigación en Emprendimiento, un reto para la Construcción de Conocimiento. Revista EAN.

Matiz Francisco (2010). La Financiación de Nuevas Empresas, el caso colombiano. Coyuntura Pyme $\mathrm{N}^{\circ} 32$

Matiz F; Mogollón Y (2008). La cadena de financiación: Una necesidad para el desarrollo económico y social a partir del emprendimiento. Revista EAN.

Postigo S. et al. (2002). El surgimiento de emprendedores de base universitaria: ¿En qué se diferencian?

Van Praag, C.M. y Cramer, J.S. (2001). An estimated equilibrium model of business formation and labour demand by entrepreneurs. Económica.

Watson, K.; Hogarth-Scott, S. y Wilson, N. (1998). Small business start-ups: success factors and support implications. International Journal of Entrepreneurial Behaviour \& Research 


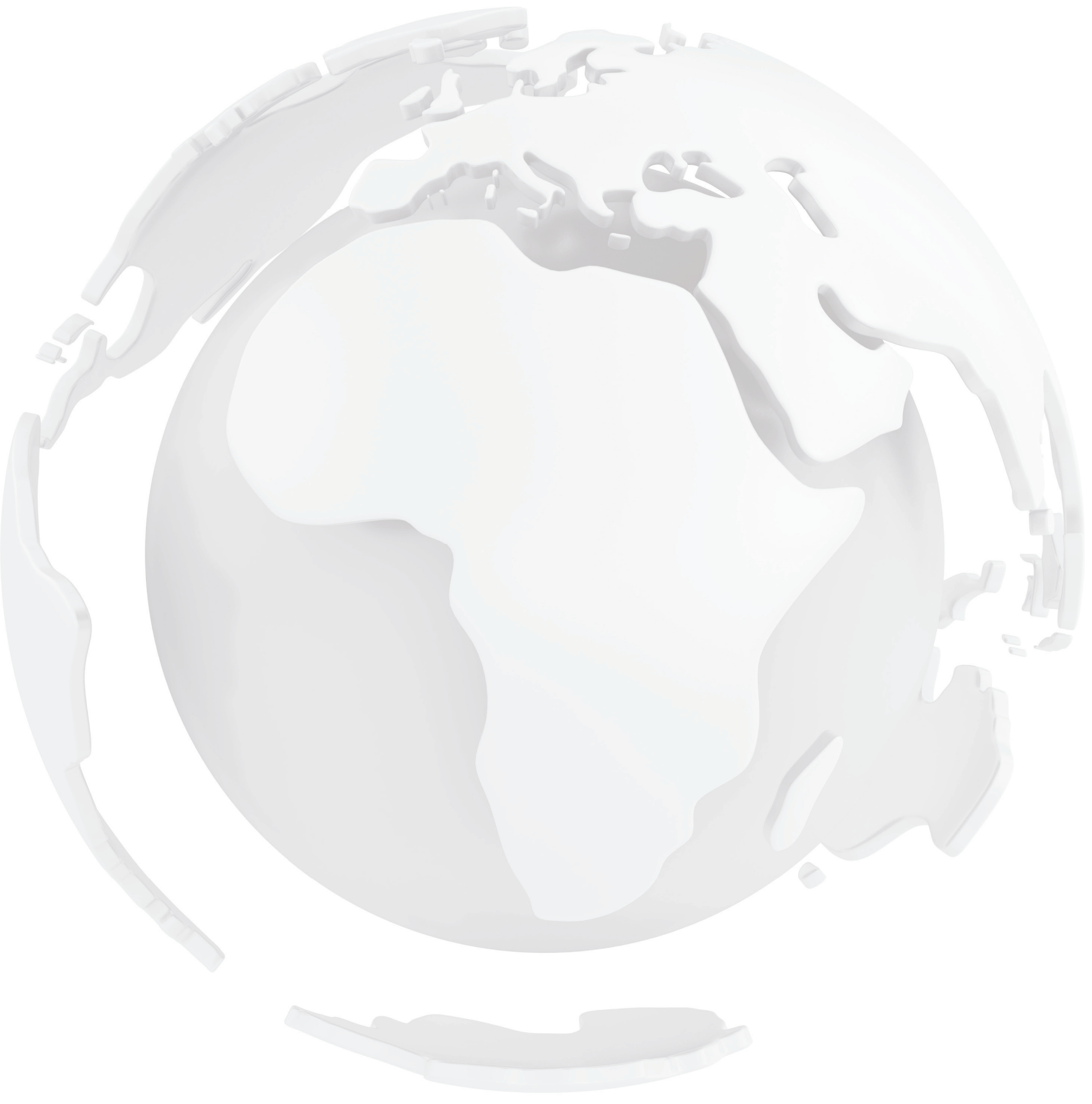

